\title{
Conformal Fabrication of an Electrospun Nanofiber Mat on a 3d Ear Cartilage-shaped Hydrogel Collector Based on Hydrogel-Assisted Electrospinning
}

Jin Yeong Song

Pusan National University

Hyun II Ryu

Pusan National University

Jeong Myeong Lee

Pusan National University

Seong Hwan Bae

Pusan National University School of Medicine Jae Woo Lee

Pusan National University School of Medicine

Changryul Claud Yi

Pusan National University School of Medicine

Sang Min Park ( $\nabla$ sangmin.park@pusan.ac.kr)

Pusan National University https://orcid.org/0000-0002-2496-4141

\section{Nano Express}

Keywords: Electrospinning, nanofiber, hydrogel, 3D Printing, ear cartilage

Posted Date: March 17th, 2021

DOl: https://doi.org/10.21203/rs.3.rs-291417/v1

License: (c) (i) This work is licensed under a Creative Commons Attribution 4.0 International License. Read Full License

Version of Record: A version of this preprint was published at Nanoscale Research Letters on July 9th, 2021. See the published version at https://doi.org/10.1186/s11671-021-03571-6. 


\section{Conformal fabrication of an electrospun nanofiber}

\section{mat on a 3D ear cartilage-shaped hydrogel}

collector based on hydrogel-assisted

\section{electrospinning}

Jin Yeong Song ${ }^{1}$, Hyun Il Ryu ${ }^{1}$, Jeong Myeong Lee ${ }^{1}$, Seong Hwan Bae ${ }^{2,3}$, Jae Woo Lee ${ }^{2}$,

Changryul Claud $\mathrm{Yi}^{2,3, * *}$, Sang Min Park ${ }^{1, *}$

${ }^{1}$ School of Mechanical Engineering, Pusan National University, 2, Busandaehak-ro 63 beongil, Geumjeong-gu, Busan, 46241, South Korea

${ }^{2}$ Department of Plastic and Reconstructive Surgery, Pusan National University School of Medicine, 179 Gudeok-ro, Seo-gu, Busan 49241, South Korea

${ }^{3}$ Biomedical Research Institute, Pusan National University Hospital, 179 Gudeok-ro, Seo-gu, Busan 49241, South Korea

\section{Corresponding Author}

*Sang Min Park - sangmin.park@pusan.ac.kr

** Changryul Claud Yi - fuff10000@gmail.com 
Keywords: Electrospinning; nanofiber; hydrogel; 3D Printing; ear cartilage

\section{ABSTRACT}

Electrospinning is a common and versatile process to produce nanofibers and deposit them on a collector as a two-dimensional nanofiber mat or a three-dimensional (3D) macroscopic arrangement. However, 3D electroconductive collectors with complex geometries, including protruded, curved, and recessed regions, generally caused hampering of a conformal deposition and incomplete covering of electrospun nanofibers. In this study, we suggested a conformal fabrication of an electrospun nanofiber mat on a 3D ear cartilage-shaped hydrogel collector based on hydrogel-assisted electrospinning. To relieve the influence of the complex geometries, we flattened the protruded parts of the 3D ear cartilage-shaped hydrogel collector by exploiting the flexibility of the hydrogel. We found that the suggested fabrication technique could significantly decrease an unevenly focused electric field, caused by the complex geometries of the 3D collector, by alleviating the standard deviation by more than $70 \%$ through numerical simulation. Furthermore, it was experimentally confirmed that an electrospun nanofiber mat conformally covered the flattened hydrogel collector with a uniform thickness, which was not achieved with the original hydrogel collector. Given that this study established the conformal electrospinning technique on $3 \mathrm{D}$ electroconductive collectors, it will contribute to various studies related to electrospinning, including tissue engineering, drug/cell delivery, environmental filter, and clothing. 


\section{Introduction}

Electrospinning is an effective and versatile technique for fabricating nanofibers and their assemblies, which has been extensively studied over the past decades.[1] Due to their unique properties such as high porosity, high surface to volume ratio, and extracellular matrixmimicking structure,[2] extraordinarily electromagnetism, electrospun nanofibers and their assemblies have been created substantial interests from various research fields, including clothing,[3] environmental filter,[4-6] battery,[7] and tissue-engineered scaffolds.[8-10] However, the chaotic motion of electrospun nanofibers due to bending instability has hampered the accurate and precise control in constructing nanofiber assemblies and generally created randomly interwoven 2D nanofiber mat.[11] Recent advances in the electrospinning process have expanded the range of the nanofiber assemblies from a $2 \mathrm{D}$ flat nanofiber mat to a $3 \mathrm{D}$ nanofibrous macrostructure.[12] With 3D electrospinning techniques, various 3D nanofibrous macrostructures have been fabricated, including a hollow tubular shape,[13] molded 3D complex geometries,[14] and native tissue shaped nanofibrous scaffolds.[15] Such 3D nanofibrous macrostructures have become a growing topic of interest, particularly in tissue engineering, due to not only possessing a biomimetic nanofibrous structure but also mimicking a 3D macrostructure of native tissues.[16, 17] As a result, various researches have led to an improvement in the aesthetic and performance functions of 3D nanofiber assemblies.[18, 19]

Among various 3D electrospinning techniques, including the hydrolysis, gas-forming techniques, rolling, folding, and 3D electroconductive collector, electrospinning on a 3D electroconductive collector provided a facile way to assembly electrospun nanofibers into a 3D nanofibrous macrostructure without any post-processing.[20-23] The complex geometries of the $3 \mathrm{D}$ electroconductive collector, such as a large bending or a recessed shape, enabled to 
produce aligned nanofiber mats or fluffy 3D nanofibrous macrostructures.[24] However, such complex geometries, at the same time, hampered the deposition of electrospun nanofibers on the recessed region of the collector and caused incomplete covering of the 3D electroconductive collector. Thus, it has been great difficulty to replicate the complex geometries of the 3D electroconductive collector to a 3D nanofiber mat. In this regard, it is necessary to develop a technique that replicates the shape of a 3D electroconductive collector with high complexity into a 3D nanofibrous macrostructure to broaden the application of electrospun nanofiber assemblies.

In this study, we proposed a conformal fabrication of an electrospun nanofiber mat that replicates the shape of a 3D electroconductive collector with complex geometries. As a representative example of the 3D electroconductive collector, we selected a 3D ear cartilageshaped collector for the conformal fabrication of an electrospun nanofiber mat due to its complex geometries. In the previous study, we suggested a hydrogel, which possessed sufficient mobile ions to generate the electric field like a metal collector, as an electroconductive collector for electrospinning.[25] Here, we utilized the flexibility of a hydrogel collector to conformally and uniformly deposit electrospun nanofibers on the $3 \mathrm{D}$ ear cartilage-shaped hydrogel collector. Unlike a metal collector, the hydrogel collector could be flattened due to the flexibility of the hydrogel, and thereby, generate a uniform electric field to evenly deposit nanofibers on an entire surface of the 3D ear cartilage-shaped hydrogel collector. Furthermore, the mechanical strength of the hydrogel could be adjusted to that of the native-tissue by finding an appropriate hydrogel. We selected alginate and gelatin as hydrogel materials due to their excellent biocompatibility.[26, 27] By mixing alginate and gelatin, we could attain mechanical properties similar to the native ear cartilage.[28] We numerically investigated an influence of the flattening of the 3D ear cartilage-shaped hydrogel collector on the conformal fabrication of an electrospun nanofiber mat on the collector. Finally, we 
confirmed the conformal fabrication of an electrospun nanofiber mat on the 3D ear cartilageshaped hydrogel collector by measuring the thickness of the electrospun nanofiber mat at several positions, including helix, antihelix, scapha, and antitragus.

2. Materials and methods

\subsection{Materials}

Sylgard ${ }^{\circledR} 184$ silicone elastomer base of polydimethylsiloxane (PDMS) monomer and sylgard ${ }^{\circledR} 184$ silicone elastomer hardener of the curing agent were purchased from Dow Corning (USA). Polylactic acid (PLA) filament was obtained from Snapmaker (USA). Gelatin from bovine skin, sodium salt of alginate acid, calcium chloride dihydrate $(\geq 99 \%)$, polycaprolactone (PCL, Mw 80000), and chloroform ( $\geq 99.5 \%)$ were prepared from Sigma Aldrich (USA). Deionized water and methyl alcohol ( $\geq 99.5 \%)$ were obtained from Samchun Chemical Co., Ltd. (South Korea). All materials were used without additional purification.

2.2. Fabrication of a PDMS negative mold of the 3D ear cartilage-shaped template

The CAD file of the 3D ear cartilage-shaped template was obtained through the Turbosquid website [ref] and modified in 3DS Max. The 3D ear cartilage-shaped template was then printed by a 3D printer (A150, Snapmaker, USA). PDMS monomer and curing agent were mixed at the ratio of 10:1. The uncured PDMS mixture was stirred manually for 5 mins for uniform mixing and then degassed in a vacuum chamber until all visible air bubbles disappeared. When all air bubbles disappeared, the uncured PDMS mixture was poured into a disposable weighing dish, and the 3D ear cartilage-shaped template in the dish was completely immersed in the PDMS mixture. The dish was placed into the oven and cured at $50{ }^{\circ} \mathrm{C}$ for $24 \mathrm{~h}$. After curing, the dish was cut in half, and the 3D ear cartilage-shaped template was removed to obtain a PDMS negative mold. 


\subsection{Preparation of an alginate-gelatin hydrogel}

Four alginate-gelatin hydrogels were prepared with different weight ratios (Table 1). Gelatin was dissolved for $1 \mathrm{~h}$ by a magnetic stir at $300 \mathrm{rpm}$ in $50^{\circ} \mathrm{C}$ water. Alginate was then added and mixed manually for 5 mins. Then, an alginate-gelatin gel solution was poured into the PDMS negative mold. The alginate-gelatin gel solution was ionically crosslinked for $2 \mathrm{~h}$ in $10 \% \mathrm{w} / \mathrm{w}$ calcium chloride solution. After that, an alginate-gelatin hydrogel was departed from the PDMS negative mold and utilized as an electroconductive collector for electrospinning.

\subsection{Mechanical test of an alginate-gelatin hydrogel}

The alginate-gelatin hydrogel was prepared with the shape of an ASTM D638 Type IV specimen to measure mechanical properties by the mechanical test. Each of the prepared hydrogel specimens was loaded on a universal test machine (QM100S, QMESYS, South Korea). The mechanical test was conducted under a constant displacement at a speed of $10 \mathrm{~mm}$ $\min ^{-1}$. The elastic modulus and ultimate tensile strength (UTS) of the specimen were calculated from the stress-strain curve.

\subsection{Conformal fabrication of an electrospun nanofiber mat}

PCL $(7.5 \%, w / v)$ was dissolved in chloroform-methanol (3:1) with stirring over $6 \mathrm{~h}$. A PCL solution was placed in a 3-mL plastic syringe, and a syringe pump (NE-1000, New Era Pump Systems, Inc., USA) ejected the PCL solution through the metal needle with a flow rate of 0.4 $\mathrm{mL} \mathrm{h}{ }^{-1}$. The 3D ear cartilage-shaped hydrogel collector was placed on a polymethyl methacrylate (PMMA) flat substrate, and the PMMA substrate was located $20 \mathrm{~cm}$ below the metal needle. For electrospinning, a high voltage of 19 kV (HV30, NanoNC Co., Ltd., South Korea) was applied between a 23-gauge metal needle with an inner diameter of $0.6 \mathrm{~mm}$ and 
the 3D ear cartilage-shaped hydrogel collector. To conformally deposit the electrospun nanofiber mat on the entire surface of the hydrogel collector, we flattened the outer part of the 3D ear cartilage-shaped hydrogel collector to flatten the collector. Furthermore, the 3D ear cartilage-shaped hydrogel collector was flipped to also fabricate the electrospun nanofiber mat on the other surface of the 3D ear cartilage-shaped hydrogel collector.

\subsection{Characterization of an electrospun nanofiber mat}

The nanostructure of an electrospun nanofiber mat on the 3D ear cartilage-shaped hydrogel collector was observed by scanning electron microscopy (SEM; Supra 25, Carl Zeiss, Germany), and diameters of the nanofibers were measured in the SEM image by ImageJ. For the measurement of thickness, an electrospun nanofiber mat with the 3D ear cartilage-shaped hydrogel collector was immersed in the mixture of PDMS base and curing agent at a weight ratio of 10:1. And then, the PDMS with the electrospun nanofiber mat was cured in a dry oven at a moderate temperature of $50^{\circ} \mathrm{C}$ for $24 \mathrm{~h}$. The PDMS-embedded electrospun nanofiber mat was cross-sectioned, and the thickness of the electrospun nanofiber mat was measured based on the cross-sectional image captured by a microscope (Olympus BX53F2, Olympus, Japan).

\subsection{Numerical simulation}

An electric field that developed between the metal needle and the collector was numerically simulated by COMSOL Multiphysics v5.0 (COMSOL, USA) software. Three ear cartilageshaped model collectors, which were made of copper, PLA, and hydrogel, were utilized for the numerical simulation. The 3D ear cartilage-shaped collector was simplified as a 2D crosssection geometry. A metal ground wire was connected to the 3D ear cartilage-shaped collector. Other geometrical parameters were designated the actual values of the conformal electrospinning process: (1) distance between the metal needle and the model collector of 20 
$\mathrm{cm}$ and (2) applied electrical potential of $19 \mathrm{kV}$. The hydrogel collector, which was the alginate-gelatin hydrogel ionically crosslinked with $1 \mathrm{M}$ calcium chloride solution, was modeled based on the space charge density of the interstitial fluid of the hydrogel. Mobile ions in the interstitial fluid can be described by the Boltzmann equation, resulting in the space charge density $\rho(x)$, as follows [29]:

$$
\rho(\mathrm{x})=-2 e c_{0} \sinh \left(\frac{e}{k_{B} T} \emptyset(x)\right)
$$

where $e$ is the electron charge, $c_{0}$ is the electrolyte concentration, $k_{B}$ is the Boltzmann's constant, $T$ is the temperature, and $\phi$ is the electric potential. The dielectric constant of the hydrogel collector was set as 70.[30] To plot the direction of the electric field, a reference line $l(\mathrm{x})$ of $10 \mathrm{~mm}$ was drawn $10 \mathrm{~mm}$ above the recessed region of the 3D ear cartilage-shaped collector.

\subsection{Statistical analysis}

Statistical analyses were conducted by the one-way ANOVA analysis using MINITAB v17.1.0 software (MINITAB. LCC, USA). The statistically significant value $p$ was considered to be less than 0.05 .

\section{Results and Discussion}

\subsection{Hydrogel-assisted electrospinning}

Figure 1 schematizes the conformal fabrication of an electrospun nanofiber mat on the 3D ear cartilage-shaped hydrogel collector. Figure 1a shows that the 3D ear cartilage-shaped template was printed by a fused deposition modeling (FDM) 3D printer. As the conformal fabrication process utilized the 3D printer, the printed structure can be freely designed and easily changed to suit a highly complicated shape, such as an ear cartilage. Furthermore, the 
higher resolution structures could be obtained by adopting stereolithography (SLA) or digital light processing (DLP) 3D printers, which achieved better resolution by using photopolymerization compared to the FDM 3D printer. Figure 1b shows the PDMS negative mold that replicated the $3 \mathrm{D}$ ear cartilage-shaped template made by $3 \mathrm{D}$ printing. Figure $1 \mathrm{c}$ shows the alginate-gelatin hydrogel collector with the shape of ear cartilage by replicating the PDMS negative mold. Figure 1d shows the conformal fabrication of the electrospun nanofiber mat on the 3D ear cartilage-shaped hydrogel collector. When we placed the 3D ear cartilageshaped hydrogel collector on a flat substrate, the helix of the 3D ear cartilage-shaped hydrogel collector was not contacted with the flat substrate and apart from the substrate due to the complex geometries of ear cartilage, which induced height difference among helix, scapha, and antihelix of the 3D ear cartilage-shaped hydrogel collector. Generally, the height difference due to the protruded part of a $3 \mathrm{D}$ electroconductive collector prevented a conformal fabrication of an electrospun nanofiber mat on the $3 \mathrm{D}$ electroconductive collector. This is because the protruded part of the 3D electroconductive collector attracts most of the electrospun nanofibers and hamper the deposition of the nanofibers in the lower part of the $3 \mathrm{D}$ electroconductive collector.[31, 32] To reduce the influence of the complex geometries of the $3 \mathrm{D}$ ear cartilageshaped hydrogel collector, we flattened the protruded part of the 3D ear cartilage-shaped hydrogel collector to the flat substrate by exploiting the flexibility of the hydrogel. After that, by performing electrospinning on the 3D ear cartilage-shaped hydrogel collector, the electrospun nanofiber mat was deposited conformally on the entire surface of the $3 \mathrm{D}$ ear cartilage-shaped hydrogel collector.

\subsection{Fabrication and mechanical property evaluation of the alginate-gelatin hydrogel}

Figure 2a showed the 3D ear cartilage-shaped template printed with PLA filaments. PLA has sufficient mechanical properties and a high melting temperature $\left(\sim 130^{\circ} \mathrm{C}\right)$, suitable for the 
PDMS molding with maintaining the original ear shape. The PDMS negative mold was shown in Figure 2b. The PDMS was selected as a mold material due to its flexibility required for the demolding of complex geometries. The alginate-gelatin hydrogel collector in Figure 2c shows the complex structure of the ear, such as helix, scapha, and antihelix. To reveal the broad selection of the mechanical properties of the alginate-gelatin hydrogel collector, we prepared 4 specimens for the mechanical test with the different mixing ratios of alginate and gelatin. Figure $2 \mathrm{~d}$ and $2 \mathrm{e}$ show the stress-strain curve and Young's modulus, respectively, according to the ratio of the hydrogel materials. Figure $2 d$ showed that the specimen made of pure gelatin showed the lowest mechanical strength, and by increasing the content of the alginate, the mechanical strength of the alginate-gelatin mixture was linearly increased. In Figure 2e, Young's modulus of alginate-gelatin hydrogel varied from $0.04 \mathrm{MPa}$ to $5.53 \mathrm{MPa}$. For the case of pure gelatin, named A0G100, the specimen exhibited the lowest Young's modulus of 0.04 $\pm 0.01 \mathrm{MPa}$ and thus was difficult to retain its shape during electrospinning. Conversely, the specimen of pure alginate, named A100G0, had the highest Young's modulus of $5.53 \pm 0.77$ $\mathrm{MPa}$, capable of maintaining its shape during electrospinning. The A50G50 and A25G75 specimens, which are the mixture of alginate and gelatin, exhibited Young's modulus of 2.10 $\pm 0.45 \mathrm{MPa}$ and $1.35 \pm 0.03 \mathrm{MPa}$, respectively. In particular, considering the ear cartilage as a target, Young's modulus of the A25G75 specimen was within Young's modulus range $(1 \sim 2$ $\mathrm{MPa}$ ) of the native ear cartilage, which is shown as the gray region in Figure 2e. Based on these results, the ratio of $\mathrm{A} 25 \mathrm{G} 75$ was utilized for the 3D ear cartilage-shaped hydrogel collector.

3.3. Fabrication of the nanofiber mat on an alginate-gelatin hydrogel collector

To investigate the deposition behavior of electrospun nanofibers on the alginate-gelatin hydrogel collector, we performed a numerical simulation of electric fields with three types of collectors: a copper collector, a PLA collector, and an alginate-gelatin hydrogel collector. The deposition of electrospun nanofibers was generally determined by the interaction between the 
charge of the electrospun nanofibers and the electric field. In this sense, the simulation of the electric field has been utilized to understand the deposition of electrospun nanofibers on the collector. In the simulation result with the PLA collector, as shown in Figure S1b, since most of the electric field was concentrated towards the ground, it was expected that electrospun nanofibers would not be deposited on the surface of the collector. In contrast, in the simulation result with the alginate-gelatin hydrogel collector shown in Figure S1c, the electric field was focused throughout the surface of the alginate-gelatin hydrogel collector, like a copper collector (Figure S1a). From this simulation result, we expected that electrospun nanofibers would be majorly deposited on the surface of the alginate-gelatin hydrogel collector. These simulation results were confirmed by electrospinning on the PLA and alginate-gelatin hydrogel collector and comparing the thicknesses of an electrospun nanofiber mat (Figure S1d). Similar to the simulation results with the PLA collector, most of the electrospun nanofibers were placed on a grounded wire and the helix part of the PLA collector. At the locations excluding the ground wire and the helix part, electrospun nanofibers on the surface of the PLA collector was stacked in micrometer scale or less. Contrary, the thicknesses of an electrospun nanofiber mat deposited on the helix was measured for the PLA and alginate-gelatin hydrogel collector to compare the nanofiber deposition according to the type of the collector, and the thicknesses of the nanofiber mat on each collector were $3.09 \pm 0.37 \mu \mathrm{m}$ and $33.24 \pm 2.43 \mu \mathrm{m}$, respectively (Figure S1d). This result implies that the alginate-gelatin hydrogel is an effective collector for electrospun nanofibers during electrospinning. Next, we confirmed that the alginate-gelatin hydrogel collector could produce nanoscale fibers during electrospinning. The electrospun nanofiber mat on the alginate-hydrogel collector was shown in Figure $3 \mathrm{a}$ with the incomplete cover of electrospun nanofibers on the surface of the collector. Figure $3 \mathrm{~b}$ showed a magnified SEM image of electrospun nanofibers on the alginate-gelatin hydrogel collector. The average diameter of the fabricated electrospun nanofibers on the alginate-gelatin hydrogel collector was 
$564 \pm 153 \mathrm{~nm}$, and most of the nanofibers have diameters ranged from 400 to $600 \mathrm{~nm}$ in Figure $3 \mathrm{c}$.

As shown in Figure 3a, we observed that the electrospun nanofiber mat has not fully covered the 3D ear cartilage-shaped hydrogel collector, particularly on the scapha, the recessed region between the helix and antihelix. This result indicated that there were void spaces at the electrospun nanofiber mat. Also, electrospun nanofibers were not deposited conformally on the entire surface but suspended as an aligned configuration, which was observed in the case of the inclined gap method.[24] Not only for the shape of the ear cartilage, the complex geometries of the $3 \mathrm{D}$ electroconductive collector, such as protrusion or high curvature, would generally cause incomplete covering of an electrospun nanofiber mat on the surface of the collector.

3.4. Numerical simulation of conformal fabrication of an electrospun nanofiber mat on the 3D ear cartilage-shaped hydrogel collector

To achieve the conformal fabrication of an electrospun nanofiber mat on the 3D electroconductive collector, we utilized the flexibility of the hydrogel collector, which would not be generally attained by a metal collector. The flexibility of the hydrogel collector enabled it to alter the shape of the collector, thereby reducing the height difference and flattening the hydrogel collector. Firstly, we numerically confirmed the conformal fabrication of an electrospun nanofiber mat on the 3D ear cartilage-shaped hydrogel collector. Figure 4a-(i) shows the configuration of the electrospinning process with the $3 \mathrm{D}$ ear cartilage-shaped hydrogel collector. Considering that electrospun nanofibers were difficult to be deposited on the recessed region of the 3D ear cartilage-shaped hydrogel collector, we highlighted the recessed region between the helix and antihelix with a $2 \mathrm{D}$ cross-section of the $3 \mathrm{D}$ ear cartilageshaped hydrogel collector as shown in Figure 4a-(ii). The helix was inclined at an angle around 60 degrees to the bottom substrate, thereby forming the recessed region between the helix and 
antihelix. To alleviate such a recessed region, we reduced the angle by bending the helix of the 3D ear cartilage-shaped hydrogel collector by exploiting the flexibility of the hydrogel collector, unlike a metal collector. The numerical simulation results with the different bending angles of 0, 30, and 60 degrees were shown in Figure 4b-(i), 4b-(ii), and 4b-(iii), respectively. Figure $4 b$-(iv) shows the angle of the electric field along the imaginary line for three cases. The average values in the angle of the electric field with imaginary lines were $79.56,79.39$, and 77.26 degrees with the bending angles of 0,30 , and 60 degrees, respectively, showing a biased angle with no significant variation between each case. Such a biased angle was caused because the recessed region between the helix and antihelix was the left part of the 3D ear cartilageshaped hydrogel collector, as shown in Figure 4a-(i). For the case of the angle deviation of the electric field, the case of the bending angle of 0 degrees showed a deviation of 8.23 degrees along the reference line $l(\mathrm{x})$. In contrast, by bending the helix at 60 degrees, the angle deviation of the electric field greatly was reduced by 2.36 degrees, which was more than $70 \%$ alleviated from the angle deviation of the electric field caused by bending 0 degrees. Such large-angle deviation for the case of the bending angle of 0 degrees would be attributed from the focused electric field toward the protruded helix, which resulted in the concentrated deposition of electrospun nanofibers on the helix and thereby hampered the conformal fabrication of an electrospun nanofiber mat on the 3D ear cartilage-shaped hydrogel collector. The reduction of angle deviation by bending the helix relieved such concentrated electric field, and therefore, bending the helix is expected to enable conformal deposition of electrospun nanofibers on the 3D ear cartilage-shaped hydrogel collector.

3.5. Conformal fabrication of an electrospun nanofiber mat on the $3 \mathrm{D}$ ear cartilage-shaped hydrogel collector 
To achieve the conformal fabrication of an electrospun nanofiber mat on the 3D ear cartilageshaped hydrogel collector, the hydrogel collector should be bent to be flattened following the simulation result. In this study, we flattened the 3D ear cartilage-shaped hydrogel collector to bend the helix. The helix and outer parts were flattened with metal fixtures as shown in Figure S2b. After that, electrospinning was performed on the original and flattened 3D ear cartilageshaped hydrogel collector. The cross-section images of the original and flattened 3D ear cartilage-shaped hydrogel collector after electrospinning were shown in Figure 5a-(i) and 5a(ii). In the case of the original 3D ear cartilage-shaped hydrogel collector, electrospun nanofibers were suspended between helix and antihelix, not deposited on the scapha of the 3D ear cartilage-shaped hydrogel collector. When the 3D ear cartilage-shaped hydrogel collector was flattened, an electrospun nanofiber mat entirely covered the helix, scapha, and antihelix. Especially, electrospun nanofibers could be deposited on the recessed region between helix and antihelix, which was not possible without flattening the 3D ear cartilage-shaped hydrogel collector. The dramatic thickness difference between the electrospun nanofiber mat on the original and the flattened 3D ear cartilage-shaped hydrogel collector was shown in Figure 5b. The thicknesses of the electrospun nanofiber mat on the original and flattened hydrogel collector were $10.76 \pm 1.21 \mu \mathrm{m}$ and $51.49 \pm 1.24 \mu \mathrm{m}$, respectively, which allowed us to achieve about 4 times compared to the thickness of the electrospun nanofiber mat by traditional electrospinning technique. With this result, electrospun nanofibers were conformally deposited on the 3D ear cartilage-shaped hydrogel collector even with complex geometries, such as helix and antihelix, by flattening the collector by utilizing the flexibility of the hydrogel collector. Lastly, we confirmed the uniformity of an electrospun nanofiber mat on the flattened 3D ear cartilage-shaped hydrogel collector (Figure 6). Figure 6a shows that an electrospun nanofiber mat could entirely cover the 3D ear cartilage-shaped hydrogel collector without showing any voids for the case of the original 3D ear cartilage-shaped hydrogel collector, as shown in Figure 
3a. Figure $6 \mathrm{~b}$ shows the thickness of the electrospun nanofiber mat deposited at the helix $(54.58$ $\pm 3.99 \mu \mathrm{m})$, the antihelix $(55.40 \pm 1.17 \mu \mathrm{m})$, the antitragus $(53.05 \pm 1.39 \mu \mathrm{m})$, and the scapha $(51.49 \pm 1.24 \mu \mathrm{m})$, where the nanofibers were not deposited with the original 3D ear cartilageshaped hydrogel collector. As a result of the electrospinning with flattened 3D ear cartilageshaped hydrogel collector, the electrospun nanofiber mat was deposited conformally and uniformly on the hydrogel collector. This nanofiber mat on the 3D ear cartilage-shaped hydrogel collector having mechanical properties similar to those of native ear cartilage could be expected to be applied as an artificial ear cartilage implant. We believed that this conformal fabrication of an electrospun nanofiber mat is pioneering work to produce 3D nanofiber membrane, and thus, could be utilized in a broad range of applications suggesting a novel type of nanofiber assemblies such as 3D native-tissue mimicking scaffold or 3D porous membrane for efficient filtering.

\section{Conclusion.}

In summary, we developed the conformal fabrication of an electrospun nanofiber mat on the 3D ear cartilage-shaped hydrogel collector that has the recessed region. As a result, the nanofiber mat on the 3D ear cartilage-shaped hydrogel collector was produced with the fully replicated shape of the collector. Most importantly, the utilization of the flexibility of the hydrogel collector allowed to adjust the recessed region of the collector, and thereby, an electrospun nanofiber mat was conformally deposited with the uniform thickness on the entire surface of the collector which would not be covered with the conventional electrospinning. Given that this conformal fabrication technique would be compatible with a variety of hydrogel materials, it is expected to be a more versatile and effective technique for fabricating conformal nanofiber mat in the various fields of tissue engineering, drug/cell delivery, clothing, and battery. 
a

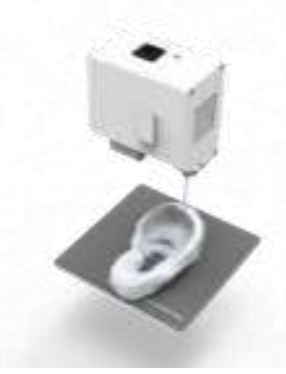

b

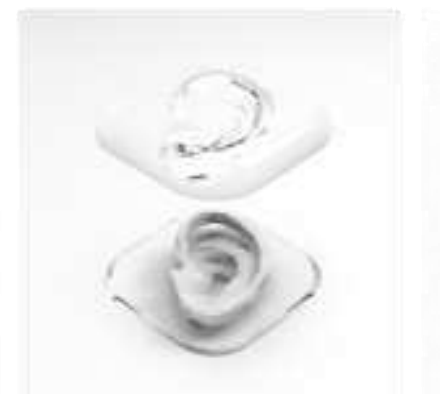

C

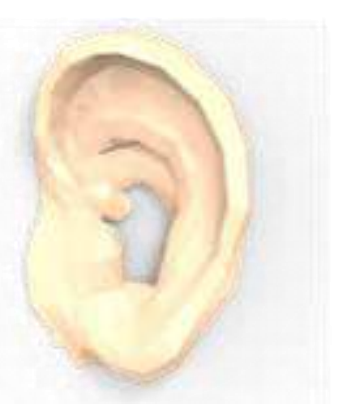

d

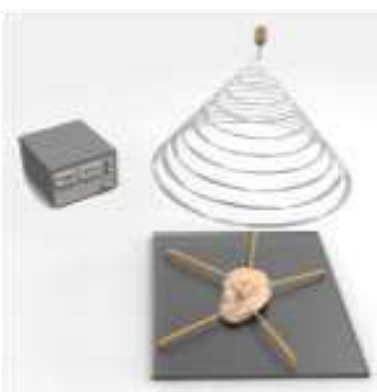

Figure 1. Schematized process of conformal fabrication of an electrospun nanofiber mat on a 3D ear cartilage-shaped hydrogel collector. (a) The 3D printing process for the 3D ear cartilageshaped template. (b) The PDMS negative mold replicated by the 3D ear-cartilage-shaped template. (c) The alginate-gelatin hydrogel collector with the shape of the ear cartilage. (d) Conformal fabrication of an electrospun nanofiber mat with the flattened 3D ear cartilageshaped hydrogel collector. 


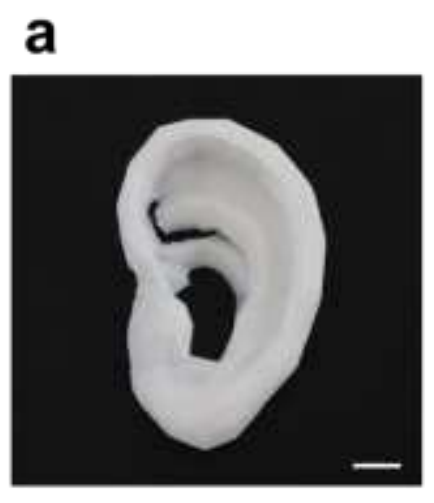

\section{d}

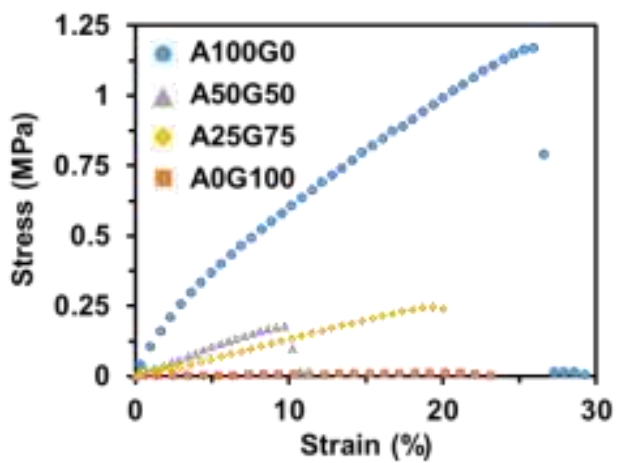

b

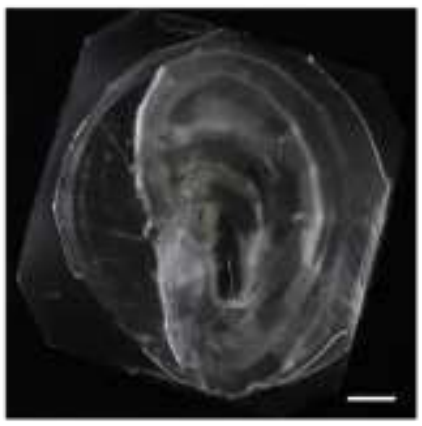

e

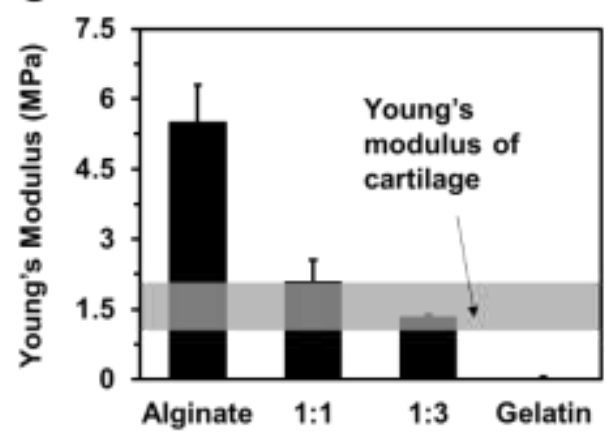

Figure 2. (a) The 3D ear cartilage-shaped template printed by an FDM 3D printer. (b) The PDMS negative mold for the production of the 3D ear cartilage-shaped hydrogel collector. (c) 3D ear cartilage-shaped hydrogel collector made of a mixture of alginate and gelatin. (d) Strainstress curve of the 4 specimens with the different mixing ratio of alginate and gelatin. (e) Young's modulus of the 4 specimens and the range of the Young's modulus of native ear cartilage (gray region). All scale bars are $1 \mathrm{~cm}$. 

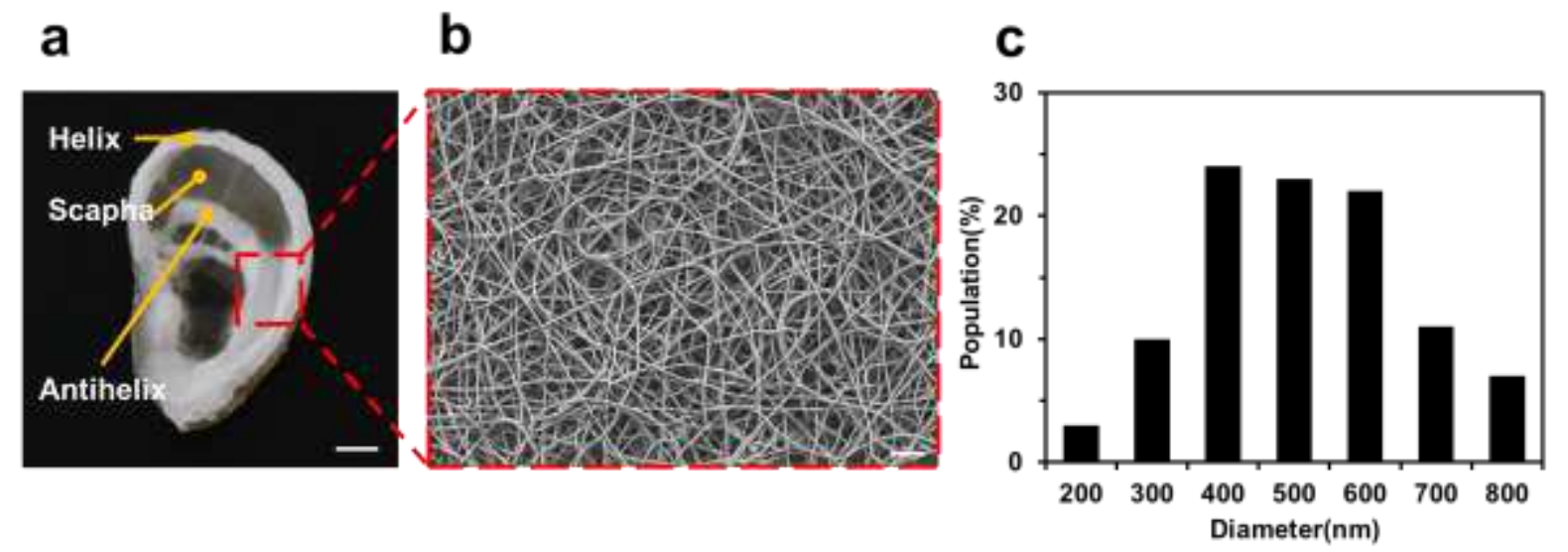

Figure 3. (a) An electrospun nanofiber mat on the 3D ear cartilage-shaped hydrogel collector.

(b) A magnified image of the electrospun nanofiber mat on the 3D ear cartilage-shaped hydrogel collector dashed in (a). (c) The population of the electrospun nanofibers on the 3D ear cartilage-shaped hydrogel collector. The scale bars are $1 \mathrm{~cm}(\mathrm{a})$ and $1 \mu \mathrm{m}(\mathrm{b})$. 

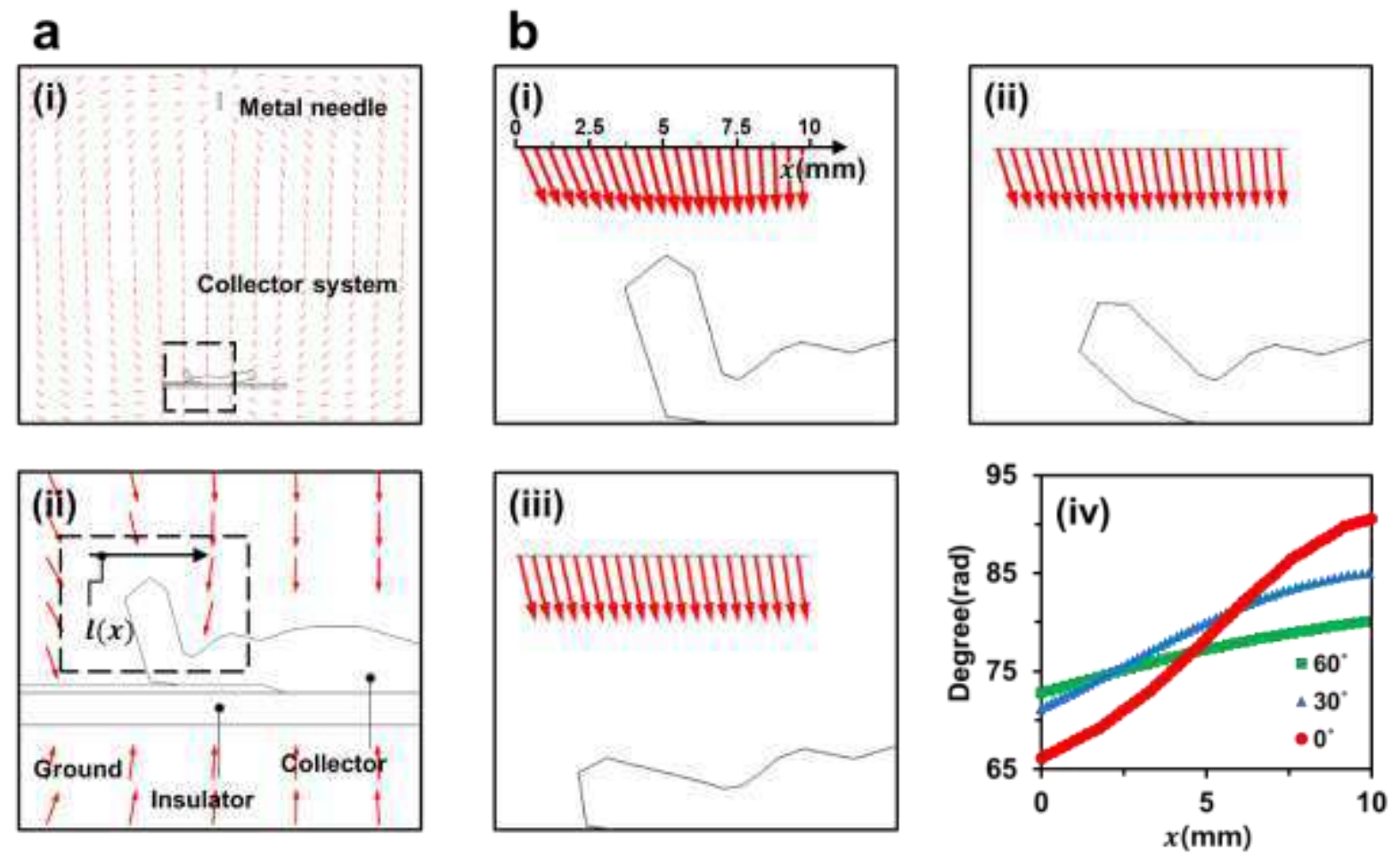

Figure 4. Electric field simulations for the hydrogel collector (a-(i)) and a magnified image of the dashed rectangular in a-(i) (a-(ii)). (b) The dashed rectangular in a-(ii) showing the recessed region of the hydrogel collector. The electric field arrows along the reference line $l(x)$ with the bending angles of 0 (b-(i)), 30 (b-(ii)), and 60 degrees (b-(iii)). (b-(iv)) The angle of the electric field to the 3D ear cartilage-shaped hydrogel collector with the bending angle of 0,30 , and 60 degrees along the reference line $l(\mathrm{x})$. 

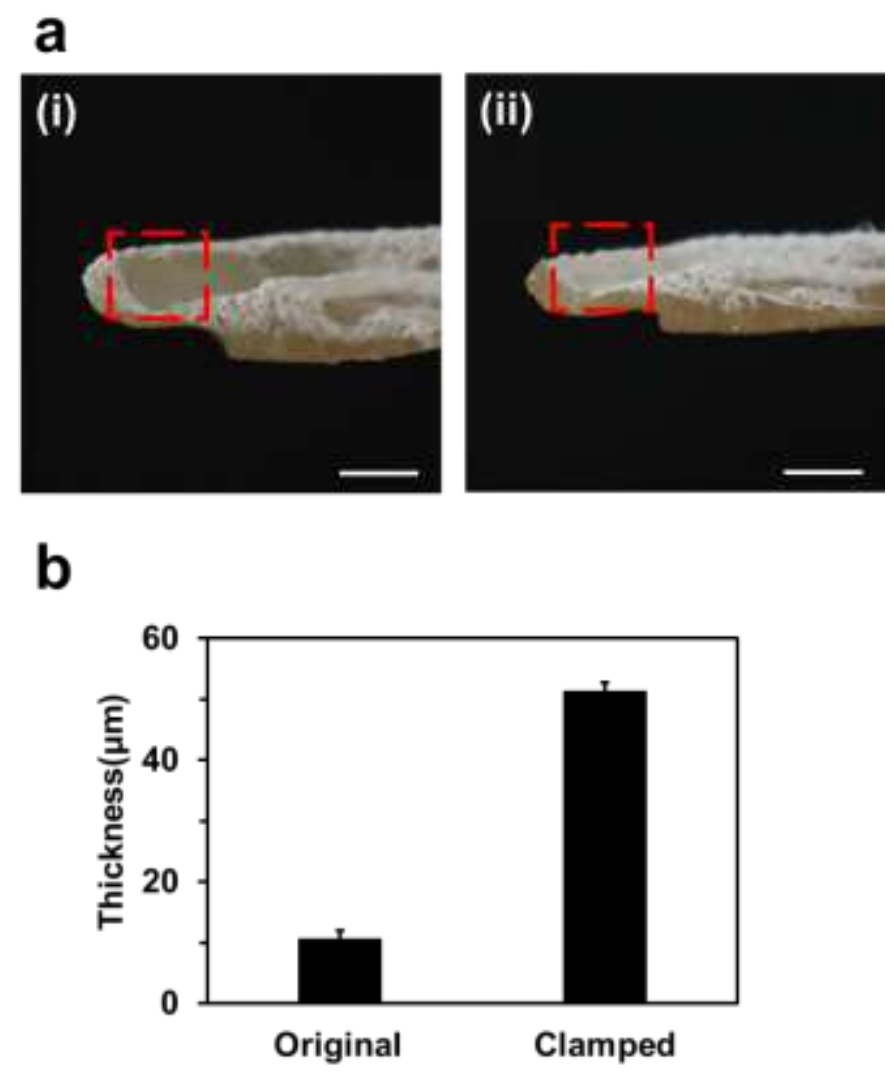

Figure 5. (a) electrospun nanofiber mats on the scapha of the original hydrogel collector (a(i)) and the flattened hydrogel collector (a-(ii)). (b) The thicknesses of the electrospun nanofiber mats at the scapha on the original and the flattened hydrogel collector. All scale bars are $1 \mathrm{~cm}$. 

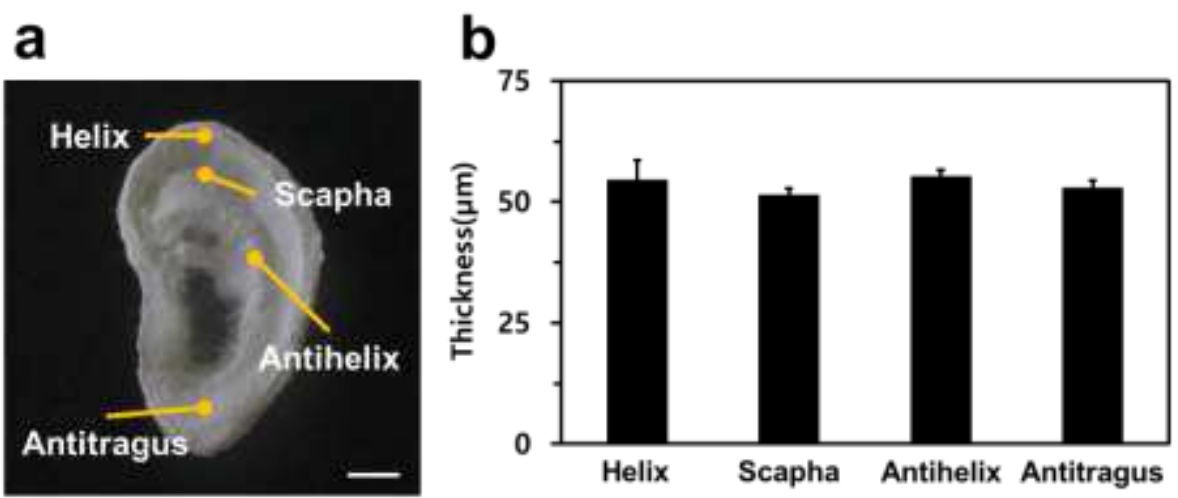

Figure 6. (a) Conformally fabricated nanofiber mat on the 3D ear cartilage-shaped hydrogel collector. The scale bar is $1 \mathrm{~cm}$. (b) The thicknesses of the electrospun nanofiber mat at several positions, including helix, scapha, antihelix, and antitragus on the flattened 3D ear cartilageshaped hydrogel collector. 
Table 1. Mixing components of the hydrogel sample

\begin{tabular}{|c|c|c|c|}
\hline Hydrogel sample & Alginate & Gelatin & Deionized water \\
\hline A0G100 & - & $3 \mathrm{~g}$ & $15 \mathrm{~g}$ \\
\hline A100G0 & $3 \mathrm{~g}$ & - & $15 \mathrm{~g}$ \\
\hline A25G75 & $0.75 \mathrm{~g}$ & $2.25 \mathrm{~g}$ & $15 \mathrm{~g}$ \\
\hline A50G50 & $1.5 \mathrm{~g}$ & & $15 \mathrm{~g}$ \\
\hline
\end{tabular}

Abbreviations: A0G100, gelatin; A100G0, alginate; A25G75, alginate : gelatin = 25:75; A50G50, alginate : gelatin $=50: 50$. 


\section{ASSOCIATED CONTENT}

Supporting Information. The following files are available free of charge.

Numerical simulation of electric fields with three types of collectors; and fabricated 3D ear cartilage-shaped alginate-gelatin hydrogel collector (PDF)

\section{AUTHOR INFORMATION}

\section{Corresponding Author}

*Sang Min Park - School of Mechanical Engineering, Pusan National University, 63-2 Busan University-ro, Geumjeong-gu, Busan, 46241, South Korea; Email: sangmin.park@pusan.ac.kr ${ }^{* *}$ Claud Changryul Yi - Department of Plastic and Reconstructive Surgery, Pusan National University School of Medicine, 179 Gudeok-ro, Seo-gu, Busan 49241, South Korea; Email: fuff10000@gmail.com

\section{Author Contributions}

J. Y. S. conducted the electric simulation, electrospinning process, created the hydrogel specimens, and wrote the manuscript. H. I. R. established the electrospinning equipment. J. M. L. modified the design of the 3D ear cartilage-shaped template. S. H. B., J. W. L., and C. C. Y. suggested the motivation of the fabrication 3D ear cartilage-shaped hydrogel and nanofiber assemblies. C. C. Y. and S. M. P. supervised and designed the overall research. All authors reviewed the manuscript.

\section{Funding Sources}


This work was supported by the National Research Foundation of Korea (NRF) grant funded by the Korea government (MSIT) (No. 2020R1C1C100944311) and the clinical research grant from Pusan National University Hospital in 2020.

\section{ACKNOWLEDGMENT}

This work was supported by the National Research Foundation of Korea (NRF) grant funded by the Korea government (MSIT) (No. 2020R1C1C100944311) and the clinical research grant from Pusan National University Hospital in 2020.

\section{ABBREVIATIONS}

3D, three-dimensional; PDMS, polydimethylsiloxane; PLA, polylactic acid; PCL, polycaprolactone; PMMA, polymethyl methacrylate; SEM, scanning electron microscopy; FDM, fused deposition modeling; SLA, stereolithography; DLP, digital light processing; A100G0, pure alginate; A0G100, pure gelatin; A50G50, alginate : gelatin $=50: 50 ;$ A25G75, alginate : gelatin $=25: 75$.

\section{NOTES}

The authors declare no competing financial interest

\section{REFERENCES}

[1] J. Xue, T. Wu, Y. Dai, Y. Xia, Electrospinning and Electrospun Nanofibers: Methods, Materials, and Applications, Chem. Rev. 119(8) (2019) 5298-5415 DOI: https://doi.org/10.1021/acs.chemrev.8b00593. 
[2] S. Sell, C. Barnes, M. Smith, M. McClure, P. Madurantakam, J. Grant, M. McManus, G. Bowlin, Extracellular matrix regenerated: tissue engineering via electrospun biomimetic nanofibers, Polym. Int. 56(11) (2007) 1349-1360 DOI: https://doi.org/10.1002/pi.2344.

[3] D. Serbezeanu, A.M. Popa, T. Stelzig, I. Sava, R.M. Rossi, G. Fortunato, Preparation and characterization of thermally stable polyimide membranes by electrospinning for protective clothing applications, Text. Res. J. 85(17) (2015) 1763-1775 DOI:

https://doi.org/10.1177/0040517515576326.

[4] H. Liu, S. Zhang, L. Liu, J. Yu, B. Ding, A Fluffy Dual-Network Structured

Nanofiber/Net Filter Enables High-Efficiency Air Filtration, Adv. Funct. Mater. 29(39) (2019) DOI: https://doi.org/10.1002/adfm.201904108.

[5] J. Xu, C. Liu, P.-C. Hsu, K. Liu, R. Zhang, Y. Liu, Y. Cui, Roll-to-Roll Transfer of Electrospun Nanofiber Film for High-Efficiency Transparent Air Filter, Nano Lett. 16(2) (2016) 1270-1275 DOI: https://doi.org/10.1021/acs.nanolett.5b04596.

[6] R.S. Barhate, S. Ramakrishna, Nanofibrous filtering media: Filtration problems and solutions from tiny materials, J. Membr. Sci. 296(1-2) (2007) 1-8 DOI:

https://doi.org/10.1016/j.memsci.2007.03.038.

[7] L. Cao, P. An, Z. Xu, J. Huang, Performance evaluation of electrospun polyimide nonwoven separators for high power lithium-ion batteries, J. Electroanal Chem. 767 (2016) 3439 DOI: https://doi.org/10.1016/j.jelechem.2016.01.041.

[8] S.M. Park, S.J. Lee, J. Lim, B.C. Kim, S.J. Han, D.S. Kim, Versatile Fabrication of Sizeand Shape-Controllable Nanofibrous Concave Microwells for Cell Spheroid Formation, ACS Appl. Mater. Interfaces 10(44) (2018) 37878-37885 DOI:

https://doi.org/10.1021/acsami.8b15821.

[9] S.M. Park, H. Kim, K.H. Song, S. Eom, H. Park, J. Doh, D.S. Kim, Ultra-thin, aligned, free-standing nanofiber membranes to recapitulate multi-layered blood vessel/tissue interface for leukocyte infiltration study, Biomaterials 169 (2018) 22-34 DOI:

https://doi.org/10.1016/j.biomaterials.2018.03.053.

[10] S.M. Park, S. Eom, H. Hong, J. Yoon, S.J. Lee, B.C. Kim, H.W. Kim, D.S. Kim, Reconstruction of in vivo-like in vitro model: Enabling technologies of microfluidic systems for dynamic biochemical/mechanical stimuli, Microelectron. Eng. 203-204 (2019) 6-24 DOI: https://doi.org/10.1016/j.mee.2018.10.010.

[11] D. Li, Y. Xia, Electrospinning of nanofibers:Reinventing the wheel?, Adv. Mater. 16(14) (2004) 1151-1170 DOI: https://doi.org/10.1002/adma.200400719.

[12] B. Sun, Y.Z. Long, H.D. Zhang, M.M. Li, J.L. Duvail, X.Y. Jiang, H.L. Yin, Advances in three-dimensional nanofibrous macrostructures via electrospinning, Prog. Polym. Sci. 39(5) (2014) 862-890 DOI: https://doi.org/10.1016/j.progpolymsci.2013.06.002.

[13] S. Homaeigohar, Y. Davoudpour, Y. Habibi, M. Elbahri, The Electrospun Ceramic Hollow Nanofibers, Nanomaterials 7(11) (2017) DOI:

https://doi.org/10.3390/nano7110383.

[14] Q. Gao, H. Gu, P. Zhao, C. Zhang, M. Cao, J. Fu, Y. He, Fabrication of electrospun nanofibrous scaffolds with 3D controllable geometric shapes, Mater. Des. 157 (2018) 159-

169 DOI: https://doi.org/10.1016/j.matdes.2018.07.042.

[15] J.M. Holzwarth, P.X. Ma, 3D nanofibrous scaffolds for tissue engineering, J. Mater. Chem. 21(28) (2011) 10243-10251 DOI: https://doi.org/10.1039/C1JM10522A.

[16] X. Wang, B. Ding, B. Li, Biomimetic electrospun nanofibrous structures for tissue engineering, Mater. Today 16(6) (2013) 229-241 DOI:

https://doi.org/10.1016/j.mattod.2013.06.005. 
[17] A. De Mori, M. Pena Fernandez, G. Blunn, G. Tozzi, M. Roldo, 3D Printing and Electrospinning of Composite Hydrogels for Cartilage and Bone Tissue Engineering, Polymers 10(3) (2018) DOI: https://doi.org/10.3390/polym10030285.

[18] G.Z. Tan, Y. Zhou, Tunable 3D Nanofiber Architecture of Polycaprolactone by Divergence Electrospinning for Potential Tissue Engineering Applications, Nano-Micro Lett. 10(4) (2018) 73 DOI: https://doi.org/10.1007/s40820-018-0226-0.

[19] J. Song, G. Zhu, H. Gao, L. Wang, N. Li, X. Shi, Y. Wang, Origami meets electrospinning: a new strategy for 3D nanofiber scaffolds, Bio-Design Manuf. 1(4) (2018) 254-264 DOI: https://doi.org/10.1007/s42242-018-0027-9.

[20] J.-C. Park, T. Ito, K.-O. Kim, K.-W. Kim, B.-S. Kim, M.-S. Khil, H.-Y. Kim, I.-S. Kim, Electrospun poly(vinyl alcohol) nanofibers: effects of degree of hydrolysis and enhanced water stability, Polym. J. 42(3) (2010) 273-276 DOI: https://doi.org/10.1038/pj.2009.340. [21] Y. Chen, M. Shafiq, M. Liu, Y. Morsi, X. Mo, Advanced fabrication for electrospun three-dimensional nanofiber aerogels and scaffolds, Bioact. Mater. 5(4) (2020) 963-979 DOI: https://doi.org/10.1016/j.bioactmat.2020.06.023.

[22] T.I. Hwang, B. Maharjan, A.P. Tiwari, S. Lee, M.K. Joshi, C.H. Park, C.S. Kim, Facile fabrication of spongy nanofibrous scaffold for tissue engineering applications, Mater. Lett. 219 (2018) 119-122 DOI: https://doi.org/10.1016/j.matlet.2018.02.040.

[23] H. Xu, H. Li, Q. Ke, J. Chang, An anisotropically and heterogeneously aligned patterned electrospun scaffold with tailored mechanical property and improved bioactivity for vascular tissue engineering, ACS Appl. Mater. Interfaces 7(16) (2015) 8706-18 DOI:

https://doi.org/10.1021/acsami.5b00996.

[24] S.H. Park, D.-Y. Yang, Fabrication of aligned electrospun nanofibers by inclined gap method, J. Appl. Polym. Sci. 120(3) (2011) 1800-1807 DOI:

https://doi.org/10.1002/app.33395.

[25] S. Eom, S.M. Park, H. Hong, J. Kwon, S.-R. Oh, J. Kim, D.S. Kim, Hydrogel-Assisted Electrospinning for Fabrication of a 3D Complex Tailored Nanofiber Macrostructure, ACS Appl. Mater. Interfaces 12(46) (2020) 51212-51224 DOI:

https://doi.org/10.1021/acsami.0c14438.

[26] W. Shen, Y.L. Hsieh, Biocompatible sodium alginate fibers by aqueous processing and physical crosslinking, Carbohydr. Polym. 102 (2014) 893-900 DOI:

https://doi.org/10.1016/j.carbpol.2013.10.066.

[27] J.Y. Lai, Biocompatibility of chemically cross-linked gelatin hydrogels for ophthalmic use, J. Mater. Sci. Mater. Med. 21(6) (2010) 1899-911 DOI: https://doi.org/10.1007/s10856010-4035-3.

[28] M.F. Griffin, G. O'Toole, W. Sabbagh, M. Szarko, P.E. Butler, Comparison of the compressive mechanical properties of auricular and costal cartilage from patients with microtia, J. Biomech. 103 (2020) 109688 DOI:

https://doi.org/10.1016/j.jbiomech.2020.109688.

[29] S.M. Park, S. Eom, W. Kim, D.S. Kim, Role of Grounded Liquid Collectors in Precise Patterning of Electrospun Nanofiber Mats, Langmuir : the ACS journal of surfaces and colloids 34(1) (2018) 284-290 DOI: https://doi.org/10.1021/acs.langmuir.7b03547.

[30] S.M. Park, D.S. Kim, Electrolyte-Assisted Electrospinning for a Self-Assembled, FreeStanding Nanofiber Membrane on a Curved Surface, Adv. Mater.

27(10) (2015) 1682-1687 DOI: https://doi.org/10.1002/adma.201404741. 
[31] Z. Ding, A. Salim, B. Ziaie, Selective Nanofiber Deposition through Field-Enhanced Electrospinning, Langmuir : the ACS journal of surfaces and colloids 25(17) (2009) 96489652 DOI: https://doi.org/10.1021/la901924z.

[32] D. Zhang, J. Chang, Electrospinning of Three-Dimensional Nanofibrous Tubes with Controllable Architectures, Nano Letters 8(10) (2008) 3283-3287 DOI:

https://doi.org/10.1021/n1801667s. 


\section{Figures}

a

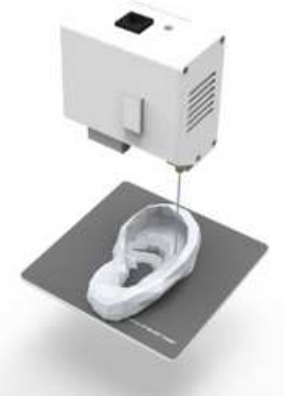

b

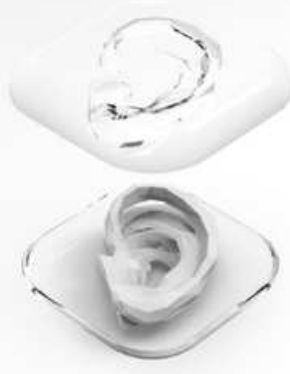

C

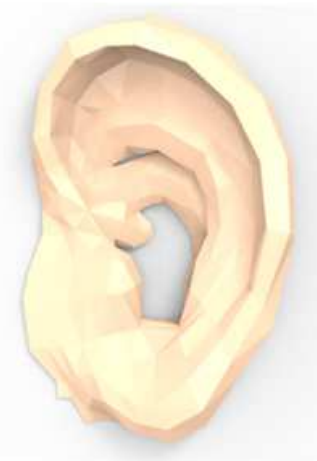

d

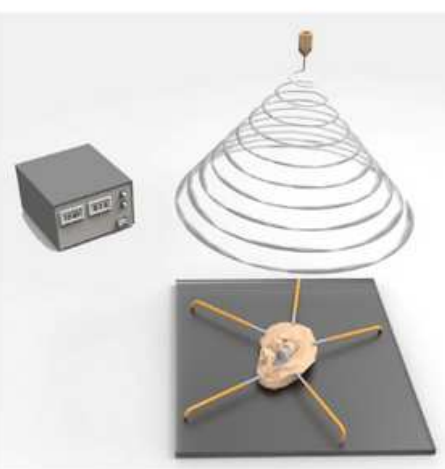

Figure 1

Schematized process of conformal fabrication of an electrospun nanofiber mat on a 3D ear cartilageshaped hydrogel collector. (a) The 3D printing process for the 3D ear cartilage-shaped template. (b) The PDMS negative mold replicated by the 3D ear-cartilage-shaped template. (c) The alginate-gelatin hydrogel collector with the shape of the ear cartilage. (d) Conformal fabrication of an electrospun nanofiber mat with the flattened 3D ear cartilage-shaped hydrogel collector. 

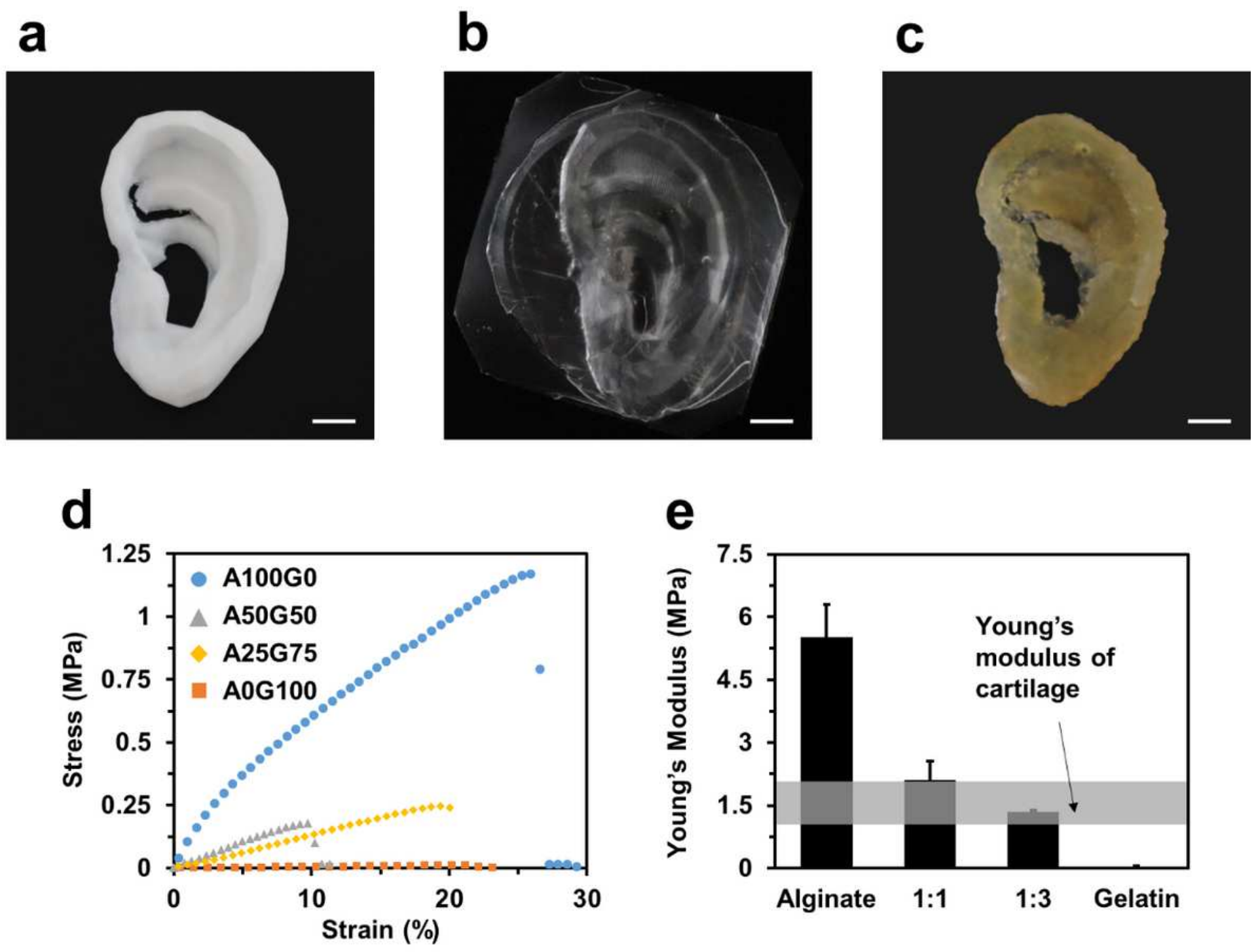

Figure 2

(a) The 3D ear cartilage-shaped template printed by an FDM 3D printer. (b) The PDMS negative mold for the production of the 3D ear cartilage-shaped hydrogel collector. (c) 3D ear cartilage-shaped hydrogel collector made of a mixture of alginate and gelatin. (d) Strain-stress curve of the 4 specimens with the different mixing ratio of alginate and gelatin. (e) Young's modulus of the 4 specimens and the range of the Young's modulus of native ear cartilage (gray region). All scale bars are $1 \mathrm{~cm}$. 

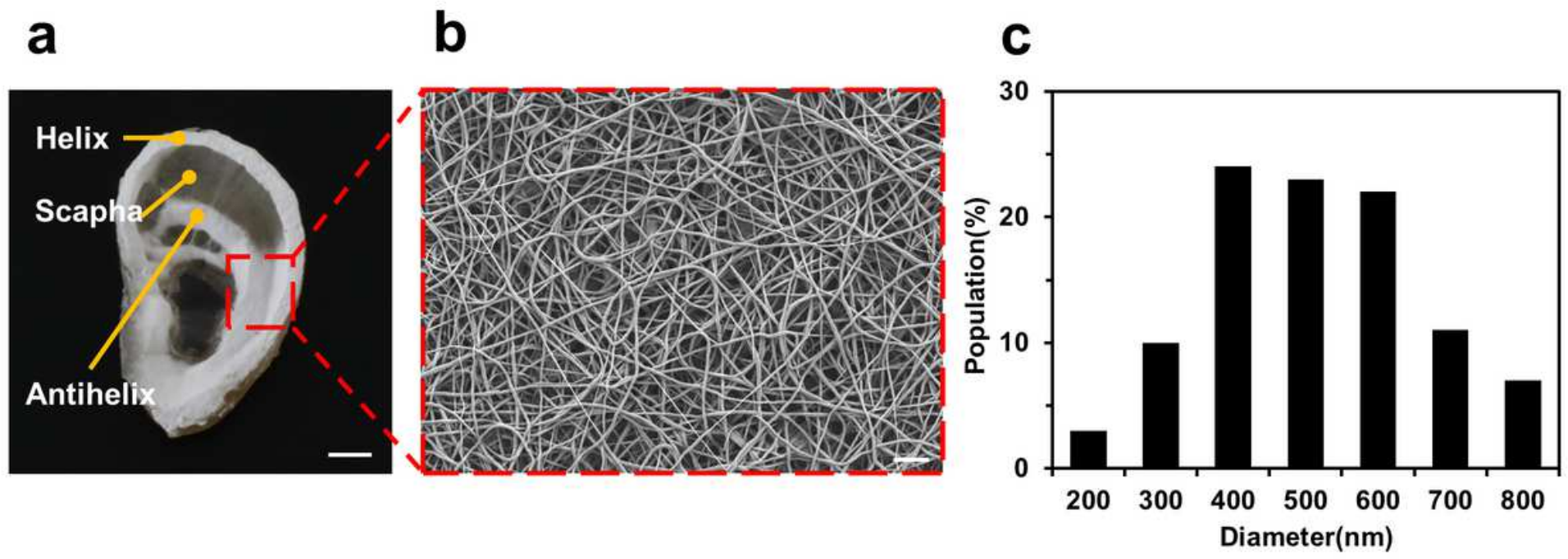

Figure 3

(a) An electrospun nanofiber mat on the 3D ear cartilage-shaped hydrogel collector. (b) A magnified image of the electrospun nanofiber mat on the 3D ear cartilage-shaped hydrogel collector dashed in (a). (c) The population of the electrospun nanofibers on the 3D ear cartilage-shaped hydrogel collector. The scale bars are $1 \mathrm{~cm}(\mathrm{a})$ and $1 \mu \mathrm{m}(\mathrm{b})$.
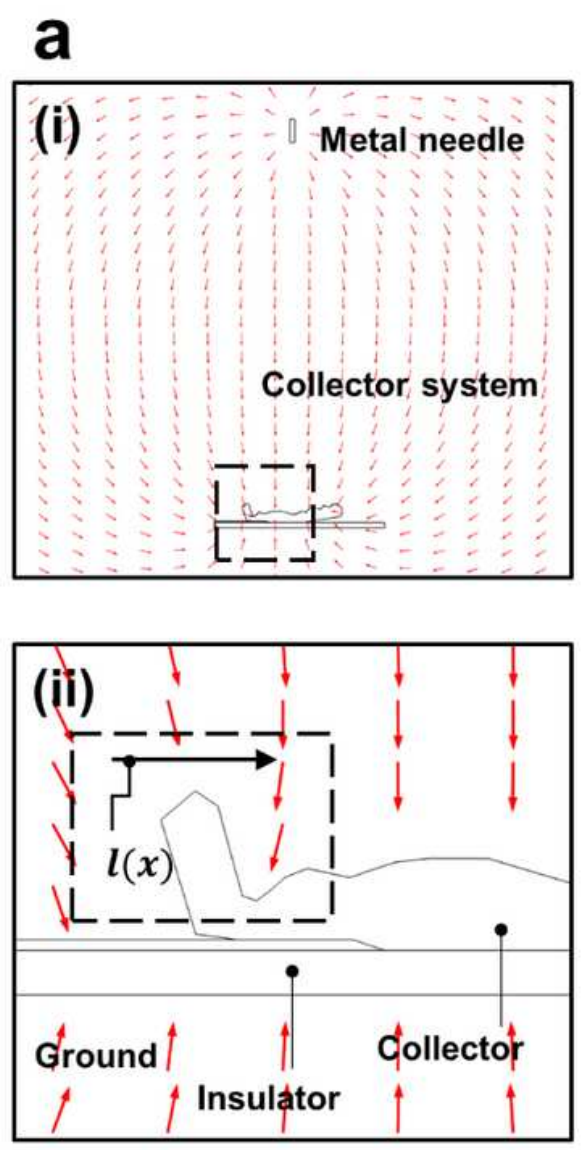

b

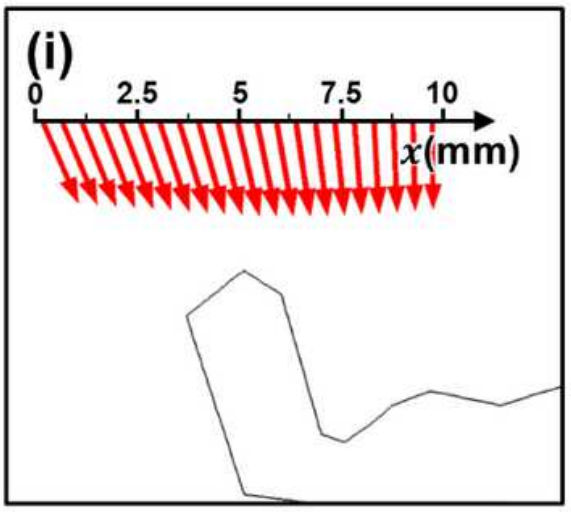

(iii)

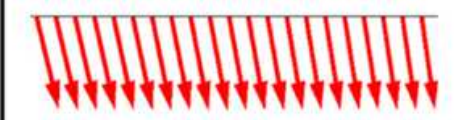

(ii)

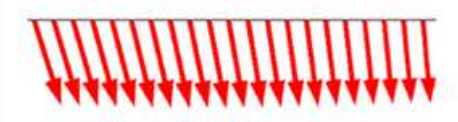

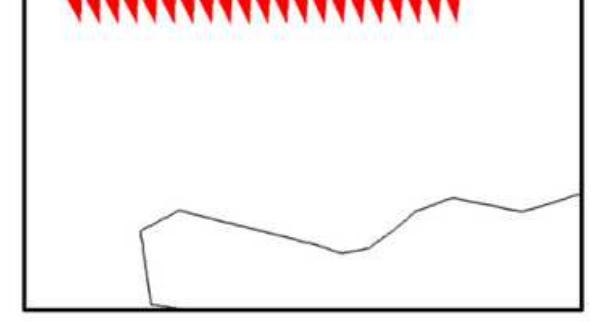


Electric field simulations for the hydrogel collector (a-(i)) and a magnified image of the dashed rectangular in a-(i) (a-(ii)). (b) The dashed rectangular in a-(ii) showing the recessed region of the hydrogel collector. The electric field arrows along the reference line $\mathrm{I}(\mathrm{x})$ with the bending angles of $0(\mathrm{~b}-(\mathrm{i})), 30$ (b(ii)), and 60 degrees (b-(iii)). (b-(iv)) The angle of the electric field to the 3D ear cartilage-shaped hydrogel collector with the bending angle of 0,30 , and 60 degrees along the reference line $\mathrm{I}(\mathrm{x})$.
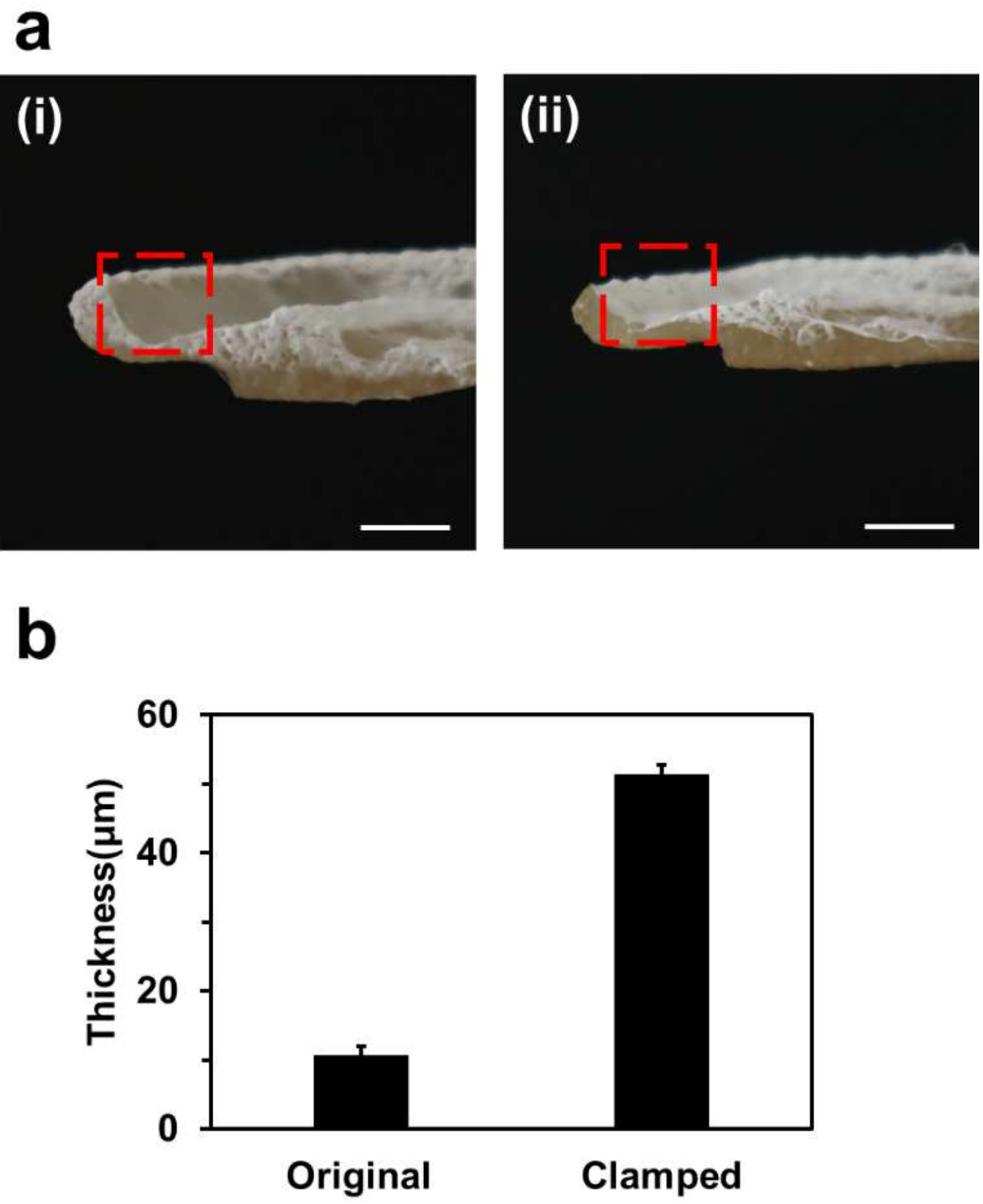

Figure 5 
(a) electrospun nanofiber mats on the scapha of the original hydrogel collector (a-(i)) and the flattened hydrogel collector (a-(ii)). (b) The thicknesses of the electrospun nanofiber mats at the scapha on the original and the flattened hydrogel collector. All scale bars are $1 \mathrm{~cm}$.
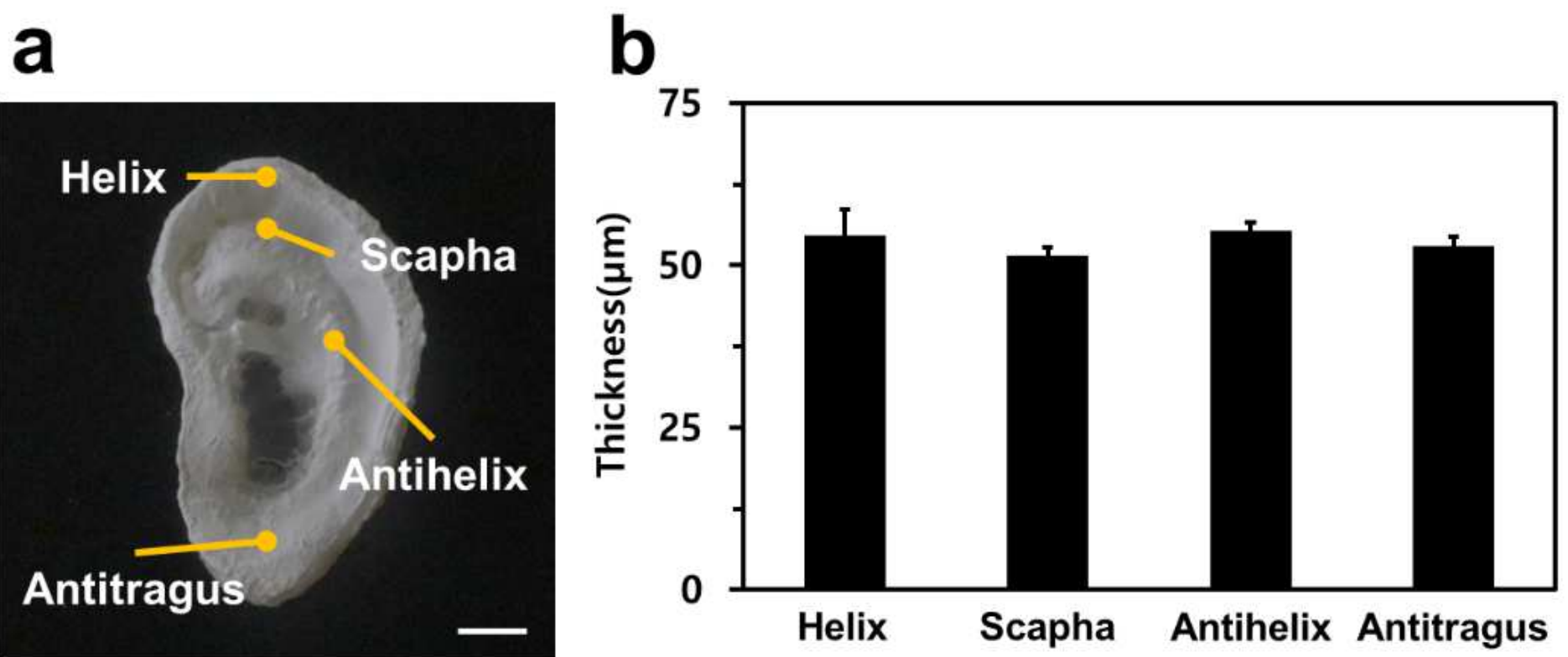

Figure 6

(a) Conformally fabricated nanofiber mat on the 3D ear cartilage-shaped hydrogel collector. The scale bar is $1 \mathrm{~cm}$. (b) The thicknesses of the electrospun nanofiber mat at several positions, including helix, scapha, antihelix, and antitragus on the flattened 3D ear cartilage-shaped hydrogel collector.

\section{Supplementary Files}

This is a list of supplementary files associated with this preprint. Click to download.

- 20210302SupportingInformationjys.docx 